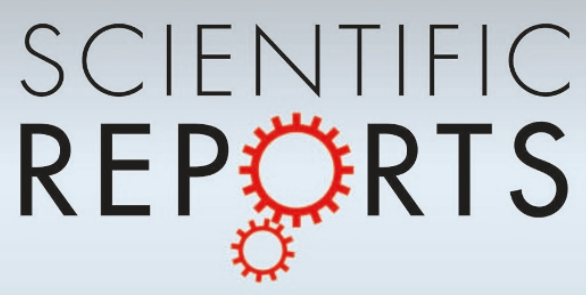

OPEN

SUBJECT AREAS:

QUANTUM OPTICS

NONLINEAR OPTICS

FIBRE OPTICS AND OPTICAL

COMMUNICATIONS

QUANTUM INFORMATION

Received

30 May 2013

Accepted

3 June 2013

Published

20 June 2013

Correspondence and requests for materials should be addressed to

A.R.M. (alex.

mcmillan@bristol.ac.

uk) or L.L. (laurent.

labonte@unice.fr)

* Current address: Currently with the GAP (University of Geneva)

\title{
Two-photon interference between disparate sources for quantum networking
}

\author{
A. R. McMillan 1,2, L. Labonté ${ }^{3}$, A. S. Clark ${ }^{1,4}$, B. Bell' , O. Alibart ${ }^{3}$, A. Martin ${ }^{3 *}$, W. J. Wadsworth'2, \\ S. Tanzilli ${ }^{3} \&$ J. G. Rarity
}

${ }^{1}$ Centre for Communications Research, Department of Electrical and Electronic Engineering, University of Bristol, Merchant Venturers Building, Woodland Road, Bristol, BS8 1UB, UK, ${ }^{2}$ Centre for Photonics and Photonic Materials, Department of Physics, University of Bath, Claverton Down, Bath, BA2 7AY, UK, ${ }^{3}$ Laboratoire de Physique de la Matière Condensée, CNRS UMR 7336, Université de Nice - Sophia Antipolis, Parc Valrose, 06108 Nice Cedex 2, France, ${ }^{4}$ Centre for Ultrahigh Bandwidth Devices for Optical Systems (CUDOS), Institute of Photonics and Optical Science (IPOS), School of Physics, University of Sydney, NSW 2006, Australia.

Quantum networks involve entanglement sharing between multiple users. Ideally, any two users would be able to connect regardless of the type of photon source they employ, provided they fulfill the requirements for two-photon interference. From a theoretical perspective, photons coming from different origins can interfere with a perfect visibility, provided they are made indistinguishable in all degrees of freedom.

Previous experimental demonstrations of such a scenario have been limited to photon wavelengths below $900 \mathrm{~nm}$, unsuitable for long distance communication, and suffered from low interference visibility. We report two-photon interference using two disparate heralded single photon sources, which involve different nonlinear effects, operating in the telecom wavelength range. The measured visibility of the two-photon interference is $80 \pm 4 \%$, which paves the way to hybrid universal quantum networks.

\footnotetext{
( onnecting distant quantum devices enables quantum networking applications, such as distributed quantum computing ${ }^{1}$ and quantum key distribution $(\mathrm{QKD})^{2,3}$. Quantum networks $(\mathrm{QNs})$ mostly exploit entanglement as a primary resource distributed between various partners that are not necessarily connected by a direct link. As sketched in Fig. 1, some nodes are capable of emitting and receiving (entangled) quantum bits of information (qubits), while others are dedicated to creating or measuring entanglement. Combining different pairs of entangled qubits by a local quantum operation, as in quantum relays ${ }^{4}$ and repeaters ${ }^{5}$, allows qubits that never physically meet to become entangled. This operation is called "entanglement swapping"6 which allows, by chaining such operations, the creation of end-to-end quantum links between arbitrarily-spaced users. From the general perspective, the specific choice of the entanglement carriers and associated observables does not matter ${ }^{7}$. Atoms and ions are preferred for qubit storage and manipulation at specific locations ${ }^{8,5}$, while photons are ideal carriers for transferring qubits over relatively long distances, as well as for on-chip manipulation $^{9,10}$. Telecom wavelength photons can travel along low-loss optical fibres, with filtering and routing advantageously handled using high performance fibre components. Recent progress has identified Fourier-transform limited picosecond photons as ideal candidates for realistic QN applications ${ }^{11}$.

From the experimental side, entanglement swapping relies on two-photon interference, a purely quantum effect which occurs when two identical photons enter opposite input ports of a beam-splitter (BS). This effect, also known as coalescence, causes the two photons to exit through the same output port of the BS and therefore leads to an absence of measured coincidences, or so-called Hong-Ou-Mandel (HOM) dip, between detectors placed at the outputs of the device ${ }^{12,13}$. Here, the type of generation process does not matter, since only the produced single photon properties have to be considered for optimizing the interference visibility. Up to now, this effect has been extensively studied using various types of sources, based either on nonlinear crystals ${ }^{11,14,15}$, quantum $\operatorname{dots}^{16-18}, \mathrm{NV}$ centers in diamond $\mathrm{d}^{19}$, single atoms $\mathrm{s}^{20}$, atomic ensembles ${ }^{21}$, or trapped ions ${ }^{22}$. However, typically the two photons originate either from different sources with the same generation process, or from the same source. While it is unclear at this stage which, if any of the currently developed photonic source technologies will be proven to be the most suitable for future QNs, it may be the case that the ideal solution will encompass multiple types of sources.

We report in this article a two-photon interference experiment involving two disparate photon-pair sources based on different nonlinear generation processes, a major step toward entanglement sharing over a QN. It is very
} 


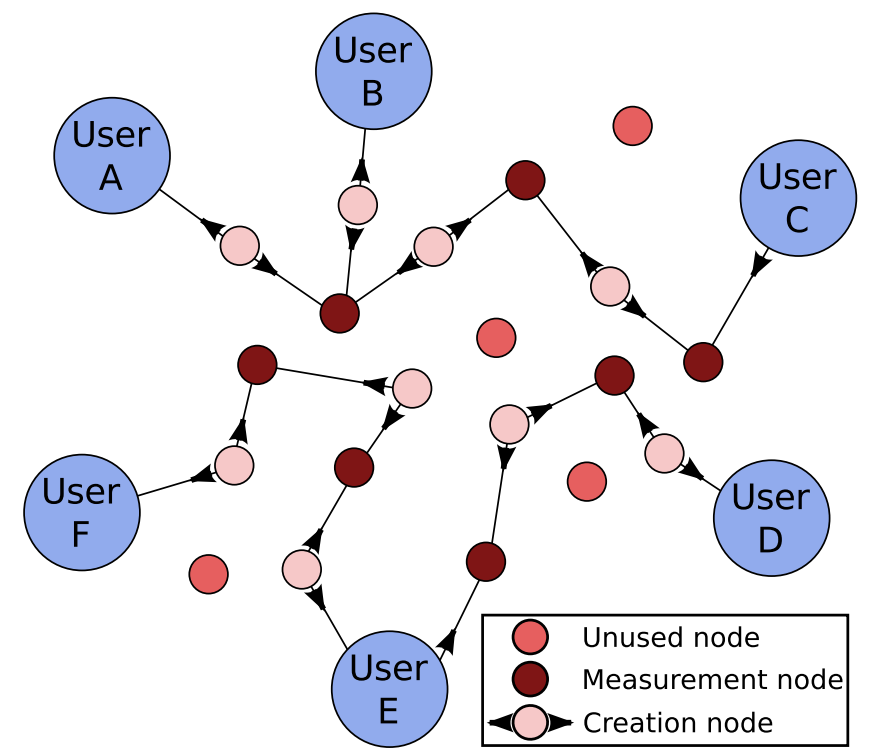

Figure $1 \mid$ Schematic of a quantum network connecting distant users through the connection of various nodes where entanglement is created or measured. The arrows define the direction to which entanglement is distributed.

recently that the first hybrid experiment has shown a measured interference visibility of $16 \%$ between two photons produced by a quantum dot and a nonlinear-crystal-based photon pair source ${ }^{23}$. This proof-of-principle experiment was limited by strong mismatches between the single photon properties, and was at an inappropriate wavelength for quantum networking. Here, we exploit photon-pair sources based on three-wave mixing in a periodically poled lithium niobate waveguide (PPLN/W) and four-wave mixing in a microstructured fiber (MF). These types of sources have already shown their capabilities in terms of pair generation efficiency and entanglement quality ${ }^{24-26}$. In the following, we describe our experimental set-up, in which photons from these two sources are made to interfere. We demonstrate a two-photon interference visibility of 80 $\pm 4 \%$ between telecom wavelength photons from these sources and discuss the relevance of this result in view of QN applications.

\section{Results}

Design and characterisation of the photon sources. Both sources have been engineered to provide paired photons emitted near $1550 \mathrm{~nm}$ and $810 \mathrm{~nm}$. The near-visible wavelength is particularly suitable for local operation since it is compatible with high efficiency detectors and quantum logic gate operations $s^{9,27,28}$. On the other hand, the telecom wavelength corresponds to the point of minimum loss in telecommunication fibre and allows standard high performance fibre components to be utilised ${ }^{11}$.

As shown in Fig. 2, two separate output beams from a picosecond pulsed, $1064 \mathrm{~nm}$ fibre laser are respectively directed towards a PPLN/W and a section of MF. Both devices are single-mode at telecom wavelengths, enabling the phase-matching of a single nonlinear process in each system and thereby avoiding any additional background noise within the bandwidth of interest. The source shown on

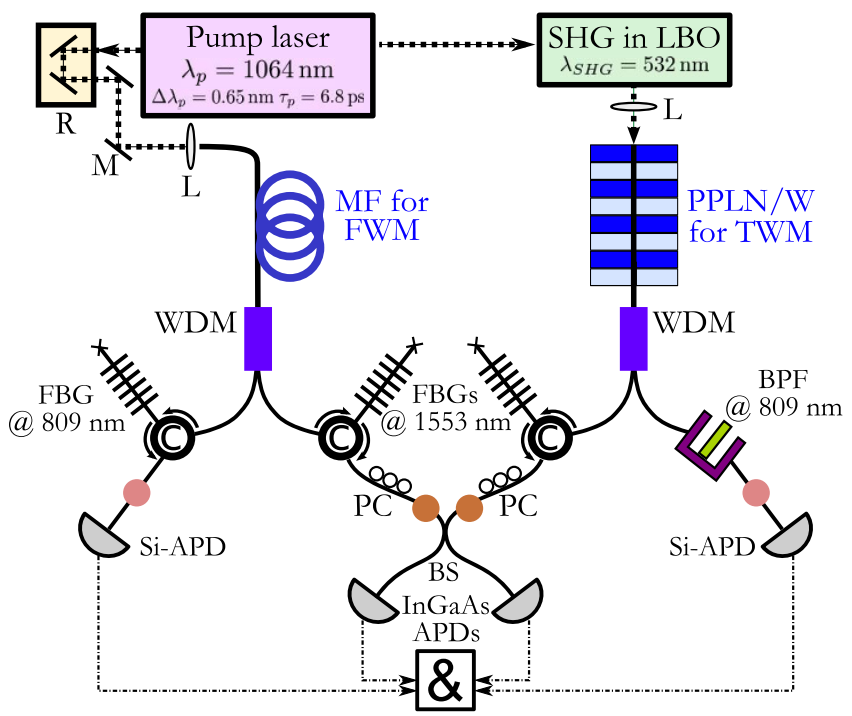

Figure $2 \mid$ Setup combining the MF-based and PPLN/W source (left and right side of diagram). The $1553 \mathrm{~nm}$ photons from both sides are filtered using FBG filters and are combined at a 50:50 fused fibre beamsplitter (BS). The two sources act as heralded single photon sources thanks to the detection of the $809 \mathrm{~nm}$ photons which herald the idler photons at $1553 \mathrm{~nm}$. R: retroreflector; M: mirror; WDM: wavelength division multiplexer; C: circulator; FBG: fibre Bragg grating filters; PC: polarization controller; APD: avalanche photodiode; \&: FPGA logic system for recording four-fold coincidences. 


\begin{tabular}{|lcc|}
\hline \multicolumn{3}{|l}{ Table 1 | Detailed operating parameters for both the MF and the } \\
PPLN/W sources & MF & PPLN/W \\
\hline \multicolumn{4}{|l}{ Sample length (cm) } & 20 & 2 \\
Idler wavelength (nm) & 1553.3 & 1553.3 \\
Signal wavelength (nm) & 809.2 & 809.2 \\
Idler FBG bandwidth (nm) & 0.6 & 0.6 \\
Signal filtering bandwidth (nm) & 0.15 & 0.5 \\
Trigger counts (kHz) & 100 & 80 \\
Coincidence counts (kHz) & 1 & 0.5 \\
Coincidence-to-accidental ratio & 20 & 20 \\
Pump power (mW) & 250 & 2 \\
\hline
\end{tabular}

the left-hand side of Fig. 2 consists of a $20 \mathrm{~cm}$ section of MF designed to exploit the $\chi^{(3)}$ nonlinearity of silica and obtain four-wave mixing (FWM), leading to paired photons near $810 / 1550 \mathrm{~nm}$ from two photons at $1064 \mathrm{~nm}^{29}$. Its length has been optimized to minimize the temporal walk-off between the pump pulse and the generated pairs of photons to obtain the best efficiency-to-bandwidth ratio. On the right-hand side, a $2 \mathrm{~cm}$-long PPLN/W fabricated through the soft-proton exchange technique ${ }^{24}$ permits photon pair production by three-wave mixing (TWM) in the $\chi^{(2)}$ nonlinear medium, at the same signal and idler wavelengths as above, upon the annihilation of a single photon at $532 \mathrm{~nm}$. In order to satisfy the requirements of energy matching for the TWM process, the initial $1064 \mathrm{~nm}$ beam is frequency doubled using a bulk lithium triborate (LBO) crystal in a temperature stabilised oven at $150^{\circ} \mathrm{C}$, before being launched into the PPLN/W.

For each source, we separate pairs of signal and idler photons into single-mode fibres using a standard wavelength demultiplexer (WDM), before applying spectral filtering in order to select energy-matched pairs of photons at wavelengths of $809.2 \mathrm{~nm}$ and $1553.3 \mathrm{~nm}$ respectively. In the case of the idler photons, which are used to demonstrate HOM interference, narrowband spectral filtering is implemented using a combination of a low loss circulator and fibre Bragg grating (FBG). These gratings are designed to reflect photons lying within a $600 \mathrm{pm}$ bandwidth, satisfying the requirements of the Fourier transform criterion for spectrally pure photons, defined by the pulse duration of the pump laser.

Both sources were characterized independently in an operating regime of a maximum of 0.05 created pairs per laser pulse. Table 1 summarizes the characteristics of these two sources. The PPLN/W exhibits a very high efficiency and consequently a pump power of $2 \mathrm{~mW}$ (at $532 \mathrm{~nm}$ ) is enough to reach the required operating regime. Furthermore, on the PPLN/W side, the emission process does not induce Raman noise, which is responsible for additional background counts in the MF source. The fibre source is inherently better adapted for coupling to standard single-mode fibres than the PPLN/W, which leads to higher coincidence count rates.

Theoretical interference visibility. The visibility $(V)$ of the HOM dip resulting from the interference of idler photons from the two sources can be written $\mathrm{as}^{30,31}$ :

$$
V=\frac{1}{\sqrt{1+\frac{\Delta t_{P P L N / W}^{2}}{2 \Delta \tau^{2}}+\frac{\Delta t_{M F}^{2}}{2 \Delta \tau^{2}}}}
$$

where $\Delta \tau$ is related to the coherence time defined by the FBG bandwidth, while $\Delta t_{P P L N / W}$ and $\Delta t_{M F}$ are the effective duration of the photon wave-packet for the PPLN/W and the MF source respectively. Note that $\Delta t$ depends on the laser pump operation regime as described by Aboussouan et al. ${ }^{11}$, and corresponds either to the detection timing jitter for the continuous wave regime, or to the idler pulse duration for the pulsed regime. Furthermore, during the propagation along the $2 \mathrm{~cm}$-long PPLN/W, the group velocity dispersion between signal $(809 \mathrm{~nm})$ and pump photons induces a 6 ps broadening of the idler photon wavepacket due to the walk-off effect. Taking into account this additional uncertainty on wavepacket arrival time, a theoretical interference visibility, given by Eq. 1, of $83 \%$ is expected.

Demonstration of two-photon interference. To maximize the interference visibility, the $1553 \mathrm{~nm}$ single photons have to be rendered indistinguishable in all their degrees of freedom. In our case, the polarization modes are made identical by using fibre polarization controllers (PC) placed just before the BS. In addition, maximal spatial mode overlap at the BS is ensured by the use of a singlemode fused fibre coupler. In order to observe HOM interference, the two photons are required to enter the BS simultaneously within their coherence time (given by the bandwidth of the idler arm FBGs). The relative arrival time of the photons at the BS is adjusted using a retroreflector $(\mathrm{R})$ mounted on a motorized translation stage, which is placed in the path of the laser pulses in front of the MF photon pair source. The signal and idler photons from both sources are detected using avalanche photodiode detectors (APD). Four-fold counts in which all four APDs detect a photon simultaneously are monitored and recorded using coincidence counting electronics (\&).

When the two idler photons are made indistinguishable, a $70 \%$ reduction in the raw four-fold coincidence rate, the HOM dip ${ }^{12}$, is demonstrated. The net four-fold coincidence rate is shown in Fig. 3, and has been corrected by subtracting the accidental background four-fold coincidence events produced by each source individually. The background contribution for each source was determined by blocking the idler arm path of the other source and counting the measured four-fold coincidence events over a period of 3.5 hours. The combined background count rate from the two sources was found to be 0.145 counts per minute, implying a total background count of 8.12 for each measured data point. The net visibility of the four-fold coincidences is $80 \pm 4 \%$, well within agreement with the expected visibility (83\%).

Moreover, the measured temporal width of the HOM dip of 16.5 \pm 2 ps agrees well with the expected value of 17 ps. We could implement narrower FBGs in order to increase $\tau_{c}$, and consequently, the visibility. Reducing the bandwidth of the idler FBGs down to $200 \mathrm{pm}$ would not have a significant impact on the counting rate, while it would increase the visibility up to $97 \%$.

\section{Discussion}

In this paper, we have implemented a set-up used to observe nonclassical HOM interference from two different types of source, an all-fibre source and a source based on a $\chi^{(2)}$ crystal. The operation of these sources relies on two different nonlinear processes (FWM and TWM). An $80 \pm 4 \%$ net interference visibility was achieved. We have identified the main factor limiting this visibility, and a reasonable way to overcome this restriction. We stress that this work will allow any pair of sources to be used together in QN applications, provided the photons generated are indistinguishable in all degrees of freedom. In this paper, the choice of the nature of the source is motivated by the need to provide proof of the feasibility of a scalable quantum network. More generally, it is the first step towards realising applications in future QNs encompassing multiple types of photon sources, such as quantum relays based on entanglement swapping operations ${ }^{32}$.

\section{Methods}

Pump source and spectral filtering. The pump laser used in this experiment was a custom dual output model, in which a single oscillator was used to provide synchronised output pulses from two separate laser amplifier stages (Fianium Ltd. FemtoPower FP-1060-0.25). Both outputs from the system supplied 7 ps-duration pulses at the wavelength of $\lambda_{p}=1064 \mathrm{~nm}$, with a spectral bandwidth of $\Delta \lambda_{p}=0.7 \mathrm{~nm}$ 


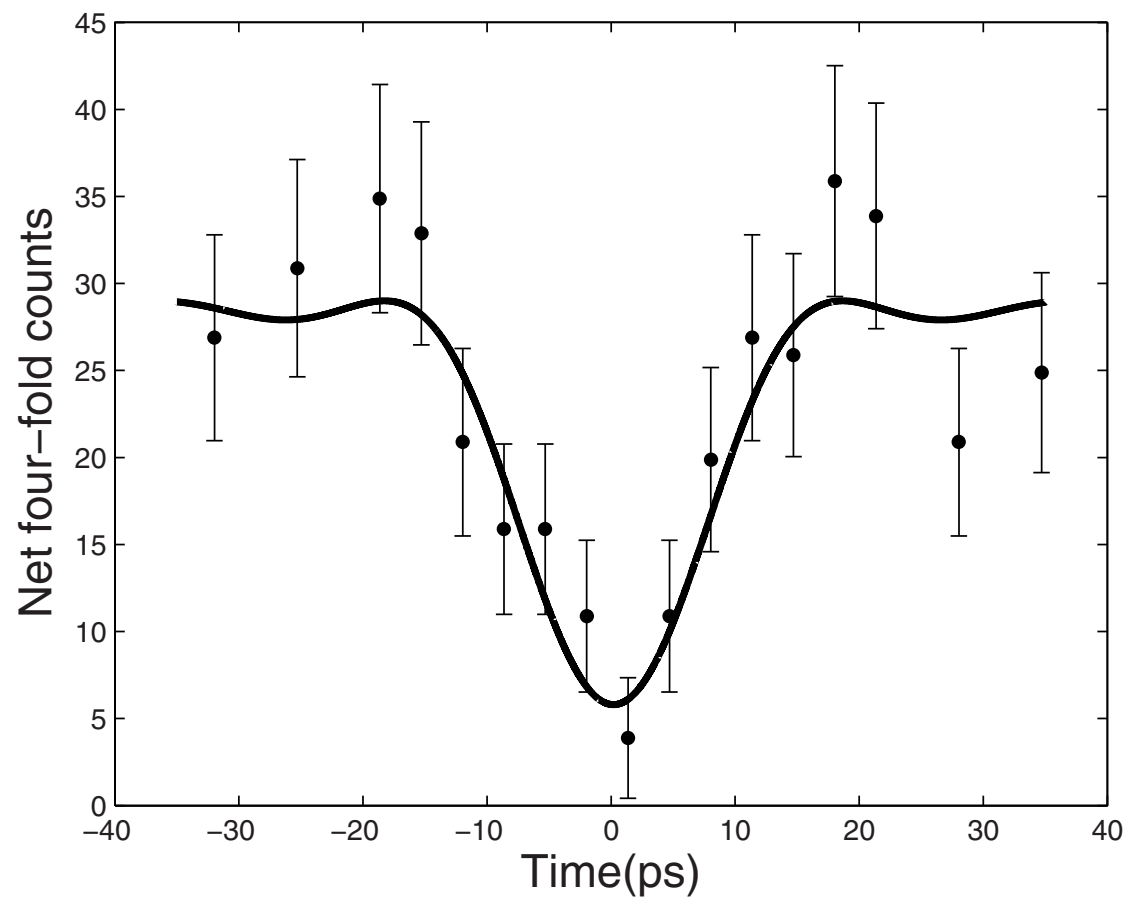

Figure $3 \mid$ Net four-fold coincidence rate as a function of the relative delay between idler photons at the BS, adjusted using the retroreflector on the MF source side. The acquisition time for data was 56 minutes at each point. The error bars are calculated based on a Poissonian distribution for the measured counts. The line shows a fit to the data, which is a sinc-squared function due to the near square shape of the spectral filtering provided by the narrowband FBGs.

and a repetition rate of $80 \mathrm{MHz}$. Based on the properties of the pump laser, $600 \mathrm{pm}$ bandwidth FBGs were selected for filtering the idler photons in both sources, corresponding to a coherence time of 13.4 ps for the interfering photons. The narrowband idler FBGs were tuned using strain to shift the central wavelength of reflection, both in order to optimally match the applied spectral filtering to the generated idler wavelengths for FWM and TWM, and to ensure that the filtering profiles of the two sources were identical. For the non-interfering signal photons in the two sources, the requirements for spectral filtering were less stringent. In the MF source, a $150 \mathrm{pm}$ bandwidth FBG was used, so that the filtering bandwidths were energy matched for both the heralding and interfering photon to help minimise the influence of uncorrelated background noise. In the PPLN/W source, where the generated noise in the idler channel was lower, the signal photons were filtered using a slightly wider $500 \mathrm{pm}$ free-space interference filter, in order to minimise the optical loss and maintain reasonable counting rates.

Detector arrangement for coincidence measurements. At the output of the set-up, four-fold photon coincidences between signal and idler photons from both sources were recorded using two sets of silicon (Si) and indium gallium arsenide (InGaAs) APD units (Perkin Elmer SPCM-AQR-14 and ID Quantique id201, respectively), all connected to a field programmable gate array (FPGA) coincidence logic system. Each InGaAs detector was advantageously gated using the heralding signal from a Si-APD after detection of an $809 \mathrm{~nm}$ photon. This allows the two systems to be operated as heralded single photon sources, and therefore limits the overall noise in the four-fold coincidence rate.

1. Grover, L. K. Quantum telecomputation. arXiv:quant-ph/9704012 (1997).

2. Gisin, N., Ribordy, G., Tittel, W. \& Zbinden, H. Quantum key distribution. J. Mod. Phys. 74, 145-195 (2002).

3. Scarani, V., Bechmann-Pasquinucci, H., Cerf, N. J., Dušek, M., Lütkenhaus, N. \& Peev, M. The security of practical quantum key distribution. Rev. Mod. Phys. 81, 1301-1350 (2009).

4. Martin, A., Alibart, O., Micheli, M. D., Ostrowsky, D. \& Tanzilli, S. A quantum relay chip based on telecommunication integrated optics technology. New J. Phys. 14, 025002 (2012)

5. Sangouard, N., Simon, C., de Riedmatten, H. \& Gisin, N. Quantum repeaters based on atomic ensembles and linear optics. Rev. Mod. Phys. 83, 33-80 (2011).

6. de Riedmatten, H., Marcikic, I., van Houwelingen, J. A. W., Tittel, W., Zbinden, H. \& Gisin, N. Longdistance entanglement swapping with photons from separated sources. Phy. Rev. A 71, 050302 (2005).

7. Tittel, W. \& Weihs, G. Photonic entanglement for fundamental tests and quantum communication. Quant. Inform. Comput. 1, 3-56 (2001).

8. Lvovsky, A. I., Sanders, B. C. \& Tittel, W. Optical quantum memory. Nature Photonics 3, 706-714 (2009)
9. Tanzilli, S., Martin, A., Kaiser, F., De Micheli, M. P., Alibart, O. \& Ostrowsky, D. B. On the genesis and evolution of integrated quantum optics. Laser Photon. Rev. 6, 115-143 (2012)

10. Martin, A., Alibart, O., Micheli, M. P. D., Ostrowsky, D. B. \& Tanzilli, S. A quantum relay chip based on telecommunication integrated optics technology. New Journal of Physics 14, 25002-25015 (2012).

11. Aboussouan, P., Alibart, O., Ostrowsky, D. B., Baldi, P. \& Tanzilli, S. Highvisibility two-photon interference at a telecom wavelength using picosecondregime separated sources. Phys. Rev. A 81, 021801(R) (2010).

12. Hong, C. K., Ou, Z. Y. \& Mandel, L. Measurement of subpicosecond time intervals between two photons by interference. Phys. Rev. Lett 59, 2044-2046 (1987).

13. Legero, T., Wilk, T., Hennrich, M., Rempe, G. \& Kuhn, A. Quantum beat of two single photons. Phys. Rev. Lett. 93, 070503 (2004).

14. Clark, A., Bell, B., Fulconis, J., Halder, M. M., Cemlyn, B., Alibart, O., Xiong, C., Wadsworth, W. J. \& Rarity, J. G. Intrinsically narrowband pair photon generation in microstructured fibres. New J. Phys. 13, 065009 (2011).

15. Kaltenbaek, R., Blauensteiner, B., Zukowski, M., Aspelmeyer, M. \& Zeilinger, A. Experimental interference of independent photons. Phys. Rev. Lett. 96, 240502 (2006).

16. Santori, C., Fattal, D., Vuckovic, J., Solomon, G. S. \& Yamamoto, Y. Indistinguishable photons from a single-photon device. Nature 419, 594-597 (2002).

17. Patel, R. B., Bennett, A. J., Farrer, I., Nicoll, C. A., Ritchie, D. A. \& Shields, A. J. Two-photon interference of the emission from electrically tunable remote quantum dots. Nature Photon. 4, 632-635 (2010).

18. Flagg, E. B., Muller, A., Polyakov, S. V., Ling, A., Migdall, A. \& Solomon, G. S. Interference of single photons from two separate semiconductor quantum dots. Phys. Rev. Lett. 104, 137401 (2010).

19. Kurtsiefer, C., Mayer, S., Zarda, P. \& Weinfurter, H. Stable solid-state source of single photons. Phys. Rev. Lett. 85, 290 (2000).

20. Beugnon, J., Jones, M. P. A., Dingjan, J., Darquié, B., Messin, G., Browaeys, A. \& Grangier, P. Quantum interference between two single photons emitted by independently trapped atoms. Nature 440, 779-782 (2006).

21. Felinto, D., Chou, C. W., Laurat, J., Schomburg, E. W., de Riedmatten, H. \& Kimble, H. J. Conditional control of the quantum states of remote atomic memories for quantum networking. Nature Physics 2, 844-848 (2006).

22. Maunz, P., Moehring, D. L., Olmschenk, S., Younge, K. C., Matsukevich, D. N. \& Monroe, C. Quantum interference of photon pairs from two remote trapped atomic ions. Nature Phys. 3, 538 (2007).

23. Polyakov, S. V., Muller, A., Flagg, E. B., Ling, A., Borjemscaia, N., Van Keuren, E., Migdall, A. \& Solomon, G. S. Coalescence of single photons emitted by disparate single-photon sources: The example of InAs quantum dots and parametric downconversion sources. Phys. Rev. Lett. 107, 157402 (2011). 
24. Tanzilli, S., Tittel, W., Riedmatten, H. D., Zbinden, H., Baldi, P., Micheli, M. D., Ostrowsky, D. \& Gisin, N. PPLN waveguide for quantum communication. Eur. Phys. J. D 18, 155-160 (2002).

25. Rarity, J., Fulconis, J., Duligall, J., Wadsworth, W. \& Russell, P. Photonic crystal fiber source of correlated photon pairs. Opt. Express 13, 534-544 (2005).

26. Fulconis, J., Alibart, O., O’Brien, J. L., Wadsworth, W. J. \& Rarity, J. G. Nonclassical interference and entanglement generation using a photonic crystal fiber pair photon source. Phys. Rev. Lett. 99, 120501 (2007)

27. O’Brien, J. L. Optical quantum computing. Science 318, 1567-1570 (2007)

28. Clark, A. S., Fulconis, J., Rarity, J. G., Wadsworth, W. J. \& O’Brien, J. L. All-opticalfiber polarization-based quantum logic gate. Phys. Rev. A 79, 030303 (Mar 2009).

29. McMillan, A. R., Fulconis, J., Halder, M., Xiong, C., Rarity, J. G. \& Wadsworth, W. J. Narrowband high-fidelity all-fibre source of heralded single photons at $1570 \mathrm{~nm}$. Opt. Express 17(8), 6156-6165 (2009).

30. Rarity, J. G. Interference of Single Photons from Separate Sources. Annals of the New York academy of Sciences 755, 624-631 (1995)

31. Zukowski, M., Zeilinger, A. \& Weinfurter, H. Entangling Photons Radiated by Independent Pulsed Sources. Annals of the New York academy of Sciences 755 91-102 (1995)

32. Halder, M., Beveratos, A., Gisin, N., Scarani, V., Simon, C. \& Zbinden, H. Entangling independent photons by time measurement. Nature Physics 3, 692-695 (2007).

\section{Acknowledgements}

The authors thank Florian Kaiser for fruitful discussions. Financial support from the Royal Society, the CNRS, the European ICT-2009.8.0 FET project QUANTIP (grant 244026), the ERC project QUOWSS (grant 247462), the EU project Q-Essence (grant 248095), the University of Bristol, the Australian Research Council Centre of Excellence (CUDOS, project number CE110001018), and the University of Nice - Sophia Antipolis, is acknowledged.

\section{Author contributions}

S.T. and J.G.R. conceived the idea for this project. The PPLN photon source was developed by L.L., O.A., A.M. and S.T., and A.R.M., W.J.W. and J.G.R. were responsible for the development of the MF source. A.R.M., L.L., A.S.C. and B.B. conducted the interference experiment. The paper was prepared by L.L., O.A. and S.T. and reviewed by all authors.

\section{Additional information}

Competing financial interests: The authors declare no competing financial interests.

How to cite this article: McMillan, A.R. et al. Two-photon interference between disparate sources for quantum networking. Sci. Rep. 3, 2032; DOI:10.1038/srep02032 (2013).

(c) (i) (5) $\odot$ This work is licensed under a Creative Commons Attribution-

NonCommercial-NoDerivs 3.0 Unported license. To view a copy of this license, visit http://creativecommons.org/licenses/by-nc-nd/3.0 